\title{
Shaping the role of germanium vacancies in germanium telluride: metastable cubic structure stabilization, band structure modification, and stable N-type conduction
}

\author{
Zihang Liu (D)', Naoki Sato', Quansheng Guo', Weihong Gao' and Takao Mori (D),2
}

\begin{abstract}
Understanding and controlling point defects in semiconductors are essential for developing advanced electronic and optoelectronic devices. Germanium telluride (GeTe), a semiconductor with a rhombohedral-to-cubic structural phase transition and a high concentration of intrinsic vacancies on the Ge sublattice, has recently attracted much interest for thermoelectric applications. However, the role of Ge vacancies in structural change and performance optimization remains obscure. Herein, we first unraveled the importance of Ge vacancies by combining first-principles calculations and Boltzmann transport theory. It is revealed that (1) Ge vacancies are more likely to spontaneously form in cubic GeTe, addressing its defective character; (2) Ge vacancies play a vital role in stabilizing cubic GeTe; and (3) Ge vacancies produce unfavorable band structure modification, leading to a reduced power factor. The following experiment found that $\mathrm{AgInTe}_{2}$ alloying promotes a symmetry change from rhombohedral to cubic and deteriorates the thermoelectric performance, in good agreement with the abovementioned conclusions. More importantly, a single-phase cubic GeTe-based material with stable n-type conduction was first discovered based on the defect chemistry approach. Our findings shed new light on the critical role of Ge vacancies in the structure-property relationship and stimulate the strategy of point defect engineering for future thermoelectric applications.
\end{abstract}

\section{Introduction}

Thermoelectric power generation (TEG), capable of directly converting heat into electricity, has received renewed interest for energy harvesting applications ${ }^{1}$, which might create a significant opportunity for future energy sustainability. The conversion efficiency of TEG is currently limited by the material's dimensionless thermoelectric figure of merit $(\mathrm{ZT}), \mathrm{ZT}=\left[S^{2} \sigma /\left(\kappa_{\text {lat }}+\kappa_{\text {ele }}\right)\right] T$, where $S, \sigma, \kappa_{\text {lat }}, \kappa_{\text {ele }}$, and $T$ are the Seebeck coefficient, electrical conductivity, lattice thermal conductivity,

Correspondence: Takao Mori (MORI.Takao@nims.go.jp)

${ }^{1}$ WPI Center for Materials Nanoarchitectonics (WPI-MANA), National Institute for Materials Science (NIMS), Namiki 1-1, Tsukuba 305-0044, Japan

${ }^{2}$ Graduate School of Pure and Applied Sciences, University of Tsukuba,

Tennodai 1-1-1, Tsukuba 305-8671, Japan

These authors contributed equally: Zihang Liu, Naoki Sato electronic thermal conductivity, and absolute temperature, respectively. In the past two decades, remarkable improvement in the $\mathrm{ZT}$ of bulk thermoelectric materials has been witnessed ${ }^{2-4}$, mainly owing to proposed new concepts or strategies, e.g., electronic structure modification to maximize the power factor $\left(\mathrm{PF}=S^{2} \sigma\right)^{5,6}$, nano-microstructural defect engineering to selectively scatter phonons ${ }^{7,8}$, strong lattice anharmonicity to identify low- $\kappa_{\text {lat }}$ materials ${ }^{9,10}$, and coupling with magnetism to decouple interrelated thermoelectric parameters ${ }^{11,12}$.

Designing chemical substitutions ${ }^{13}$, interstitials ${ }^{14,15}$, or vacancies ${ }^{16,17}$ in thermoelectrics is a conventional method to strengthen phonon scattering and therefore improve ZT. Recently, the importance of defect chemistry, both intrinsic and extrinsic, in the optimization of thermoelectric properties has been recognized ${ }^{17-19}$. For example,

\section{(c) The Author(s) 2020}

(c) Open Access This article is licensed under a Creative Commons Attribution 4.0 International License, which permits use, sharing, adaptation, distribution and reproduction c. in any medium or format, as long as you give appropriate credit to the original author(s) and the source, provide a link to the Creative Commons license, and indicate if changes were made. The images or other third party material in this article are included in the article's Creative Commons license, unless indicated otherwise in a credit line to the material. If material is not included in the article's Creative Commons license and your intended use is not permitted by statutory regulation or exceeds the permitted use, you will need to obtain permission directly from the copyright holder. To view a copy of this license, visit http://creativecommons.org/licenses/by/4.0/. 
the charge-carrier transport, e.g., the doping effectiveness, doping limit, charge-carrier mobility, and doping-site preference, is profoundly affected by the defect energy level and defect formation energy ${ }^{20}$. Since the defect chemistry in a semiconductor is dominated by the material's intrinsic properties (crystal structure and chemical composition) and external applied conditions (environment, temperature, and pressure $)^{20}$, and rational manipulation of point defects (type or concentration) to enhance the thermoelectric performance is still challenging. The most common method is tuning the sintering-process parameters ${ }^{21,22}$. Recently, incorporating extra $\mathrm{Mg}$ into Zintl phase $\mathrm{Mg}_{3} \mathrm{Sb}_{2}$ realized unusual $\mathrm{n}$-type conduction by raising the Fermi level ${ }^{19}$, which provides new insights into the development of novel thermoelectric materials.

Germanium telluride (GeTe)-based materials have attracted significant research attention for both rewriteable data storage and thermoelectric conversion applications. Similar to the abovementioned $\mathrm{Mg}_{3} \mathrm{Sb}_{2}$, GeTe exhibits persistent p-type conduction owing to the high concentration of Ge vacancies ${ }^{23}$. The microscopic mechanisms of Ge vacancies in the bonding and amorphous-crystalline phase transition of GeSbTe phase-change materials have been previously investigated by first-principles calculations. Ge vacancies annihilate energetically unfavorable antibonding $\mathrm{Ge}-\mathrm{Te}$ and $\mathrm{Sb}-\mathrm{Te}$ interactions, while vacancy redistribution and ordering drive the metal-insulator transition ${ }^{24}$. Recently, high $Z T s$ above or around 2 have continuously been reported for this system ${ }^{25-35}$. One important characteristic of pristine $\mathrm{GeTe}$ is the rhombohedral-to-cubic structural phase transition at $\sim 700 \mathrm{~K}$. High-resolution X-ray diffraction powder pattern measurements revealed that cubic GeTe possesses a larger amount of Ge vacancies than rhombohedral $\mathrm{GeTe}^{36}$. However, the majority of theoretical and experimental works on the GeTe system neglected the existence of high-concentration Ge vacancies and claimed that symmetry engineering approaching the cubic counterpart contributed to a high performance due to the more beneficial electronic band structure ${ }^{30,31,37,38}$. Therefore, it is essential to understand the true role of Ge vacancies in the crystal structure-thermoelectric property relationship. In addition, from the viewpoint of practical applications, the difficulty of designing n-type counterparts of GeTe-based materials is still a key obstacle. Recently, Samanta et al. ${ }^{39}$ first realized n-type conduction through electronic band structure modulation by $\mathrm{AgBiSe}_{2}$ alloying in GeTe.

Herein, we investigated the significance of Ge vacancies in $\mathrm{GeTe}$ based on theoretical calculations and experimental studies, which include three main parts. In the first theoretical part, the defect formation energies reveal that Ge vacancies are more likely to spontaneously form in cubic GeTe than in rhombohedral GeTe. The phonon density of states demonstrates that defective cubic GeTe with $\mathrm{Ge}$ vacancies is more dynamically stable, while
Boltzmann transport calculations reveal that the introduction of $\mathrm{Ge}$ vacancies into cubic GeTe reduces the potential power factor. In the second experimental part, $\mathrm{AgInTe}_{2}$ alloying in rhombohedral GeTe leads to the formation of cubic GeTe. All these unusual results, including the largely increased hole concentration, significantly reduced mobility, changed temperature-range dependence of the electrical resistivity, and largely suppressed lattice thermal conductivity, mean that $\mathrm{AgInTe}_{2}$ alloying spontaneously increases the concentration of $\mathrm{Ge}$ vacancies in cubic GeTe. This observation supports our previous calculation results that cubic GeTe is actually a defective structure with Ge vacancies. The deteriorated electrical properties are due to the unfavorable effect of the increased concentration of Ge vacancies, consistent with Boltzmann transport calculations. In the last experimental part, based on the obtained cubic GeTe$\mathrm{AgInTe}_{2}$ material as the parent phase, we successfully design stable $n$-type conduction by heavy Bi doping. The obtained single-phase character means that the higher solid solubility of $\mathrm{Bi}$ atoms achieved could be related to the structural difference, namely, that the highconcentration Ge vacancies in the cubic structure provide more suitable sites for foreign atoms and therefore enable a higher dopant solubility.

\section{Materials and methods Synthesis}

High-purity raw materials were directly weighed according to the nominal compositions $\mathrm{Ge}_{0.95} \mathrm{Ag}_{0.05} \mathrm{Te}$, $\mathrm{Ge}_{0.95} \mathrm{In}_{0.05} \mathrm{Te}, 0.95 \mathrm{GeTe}-0.05 \mathrm{CuInTe} \mathrm{I}_{2}, \mathrm{Ge}_{0.8} \mathrm{Bi}_{0.2} \mathrm{Te},(1-x)$ $\mathrm{GeTe}+\mathrm{xAgInTe} \mathrm{H}_{2}(x=0,0.01,0.02,0.03,0.04,0.05$, and $0.10)$, and $\left(0.95 \mathrm{GeTe}+0.05 \mathrm{AgInTe}_{2}\right)_{0.8} \mathrm{Bi}_{0.2} \mathrm{Te}$, transferred to quartz tubes, and sealed under high vacuum. These ampoules were placed vertically into a programmable resistance box furnace, heated to $1273 \mathrm{~K}$, held for $12 \mathrm{~h}$, slowly cooled to $873 \mathrm{~K}$, annealed for $72 \mathrm{~h}$, and eventually quenched to room temperature in cold water. The obtained ingots were hand ground into fine powders and consolidated by spark plasma sintering (SPS, SPS1080 System, and SPS SYNTEX INC) under a pressure of $60 \mathrm{MPa}$ at $673-773 \mathrm{~K}$ for $5 \mathrm{~min}$ to ensure a high sample density under an argon atmosphere.

\section{Phase and property characterization}

The phase structures were characterized by powder Xray diffraction (XRD, SmartLab3, Rigaku) with $\mathrm{Cu} \mathrm{K}_{\alpha}$ radiation and further analyzed by the Rietveld refinement method using FullProf. Bar samples were cut from the pressed disks and used for simultaneous measurement of the electrical resistivity $(\rho)$ and Seebeck coefficient $(S)$ on a commercial system (ULVAC ZEM-2). The thermal conductivity $\kappa_{\text {tot }}$ was calculated using $\kappa_{\text {tot }}=D C_{\mathrm{p}} d$, where $D, C_{\mathrm{p}}$, and $d$ are the thermal diffusivity, specific heat 
capacity, and density, respectively. The thermal diffusivity coefficient $(D)$ and the specific heat capacity $\left(C_{\mathrm{p}}\right)$ were concurrently measured for the disk samples on a laser flash system (Netzsch LFA 467, Germany) with a pyroceram disk as a reference sample. The sample density $(d)$ was determined by the Archimedes method. The roomtemperature Hall coefficient $R_{\mathrm{H}}$ was measured using a PPMS (Physical Properties Measurement System, Quantum Design) with the AC transport option. The data were obtained with a magnetic field sweep from $-3 \mathrm{~T}$ to $+3 \mathrm{~T}$. The Hall carrier concentration $\left(n_{\mathrm{H}}\right)$ was obtained by $n_{\mathrm{H}}=$ $1 / e R_{\mathrm{H}}$, and the Hall carrier mobility $\left(\mu_{\mathrm{H}}\right)$ was calculated by $\sigma=e \mu_{\mathrm{H}} n_{\mathrm{H}}$, where $e$ is the electronic charge and $\sigma$ is the electrical conductivity.

\section{Computational methods}

DFT total energy calculations were performed using the Vienna ab initio package (VASP) ${ }^{40}$ with projector augmented wave $(\mathrm{PAW})^{41}$ pseudopotentials. The generalized gradient approximation functional with the PerdewBurke-Ernzerhof parametrization (GGA-PBE) was chosen for the exchange and correlation potentials.

To model the defective cubic GeTe, we used the special quasirandom structure (SQS) method implemented in the Alloy Theoretical Automated Toolkit $(\mathrm{ATAT})^{42}$. We created a $3 \times 3 \times 3$ supercell with 54 atoms $\left(\mathrm{Ge}_{27} \mathrm{Te}_{27}\right.$, Supplementary Fig. S1) from the primitive cell of cubic GeTe and introduced Ge vacancies using the SQS algorithm. The modeled structures were fully relaxed without considering symmetry until the residual forces became $<10^{-3} \mathrm{eV} / \AA$. The cutoff energy was chosen as $430 \mathrm{eV}$, and a $4 \times 4 \times 4 \quad k$-point grid based on the $\Gamma$-centered Monkhorst-Pack scheme ${ }^{43}$ was used for the structure relaxation. A denser $10 \times 10 \times 10 k$-point grid was used for the calculation of the electronic density of states. The effective band structures of the defective supercells, including a cubic GeTe alloyed with $\mathrm{AgInTe}_{2}\left(\mathrm{Ge}_{62} \mathrm{~A}\right.$ $\left.\mathrm{g}_{1} \mathrm{In}_{1} \mathrm{Te}_{64}\right)$, in the primitive Brillouin zone were obtained by using the band unfolding method ${ }^{44}$. The Seebeck coefficient and the electrical conductivity were computed under the rigid band and constant relaxation time approximations using BoltzTraP code ${ }^{45}$. The calculations of the harmonic interatomic force constants and phonon density of states were performed on the defective supercells and a $4 \times 4 \times 4$ supercell based on pristine cubic GeTe using Phonopy code ${ }^{46}$.

The formation energy of defect $X$ with charge state $q$, $E^{f}\left[X^{q}\right]$, was calculated by

$$
E^{f}\left[X^{q}\right]=E_{\text {tot }}\left[X^{q}\right]-E_{\text {tot }}[\text { bulk }]-\sum_{\mathrm{i}} n_{\mathrm{i}} \mu_{\mathrm{i}}+q E_{\mathrm{F}}+E_{\text {corr }}
$$

Here, $E_{\text {tot }}\left[X^{q}\right]$ and $E_{\text {tot }}[$ bulk $]$ are the total energies of the defective and pristine supercells, respectively. We used a $4 \times 4 \times 4$ supercell for both cubic and rhombohedral
GeTe. $n_{\mathrm{i}}$ is an integer indicating the number of atoms of type $\mathrm{i}$ being added to $\left(n_{\mathrm{i}}=1\right)$ or removed from $\left(n_{\mathrm{i}}=-1\right)$ the supercell, and $\mu_{\mathrm{i}}$ is the corresponding atomic chemical potential. $E_{\mathrm{F}}$ is the Fermi energy, which serves as the chemical potential of the electron reservoir. $E_{\text {corr }}$ is a correction term that is necessary to consider the presence of periodic images within the supercell calculation. In this study, we applied the Kumagai $\operatorname{method}^{47}$ for the correction term. All procedures were performed using pycdt code ${ }^{48}$.

\section{Results and discussion}

Understanding the defect chemistry in semiconductors is a prerequisite to optimizing the physical properties, especially the thermoelectric properties. Therefore, firstprinciples calculations based on density functional theory (DFT) are performed to reveal the formation energies of the low-energy intrinsic defects, including the Te vacancy $\mathrm{Vac}_{\mathrm{Te}}$, Ge vacancy $\mathrm{Vac}_{\mathrm{Ge}}$, and antisite defects $\mathrm{Te}_{\mathrm{Ge}}$ and $\mathrm{Ge}_{\mathrm{Te}}$, in rhombohedral GeTe (R-GeTe) and cubic GeTe $(\mathrm{C}-\mathrm{GeTe})$ as a function of Fermi energy in Ge-rich and Te-rich environments (Fig. 1). Herein, the Fermi energy is referenced to the valence band maximum (VBM).

In general, the Ge vacancy exhibits the lowest defect formation energy when the Fermi level is inside the band gap. It is independent of the growth environment, basically consistent with previous calculation results of Edwards et $\mathrm{al}^{23}$. This illustrates that the Ge vacancy behaves as the most easily formed intrinsic defect in both rhombohedral and cubic GeTe. Moreover, the point at which the defect charge state changes is named the charge transition level (CTL), emphasized by the star symbols in Fig. 1. Since the CTLs of the Ge vacancy are invariably around the VBM, this means that the Ge vacancy should be a shallow acceptor, leading to a persistently high hole concentration and metallic p-type conduction. Some differences can be noted by comparing the formation energies of the Ge vacancy in the four panels of Fig. 1. First, a relatively low formation energy of the Ge vacancy is observed in the Te-rich environment, which is a common phenomenon of defect chemistry in the semiconductor area. Second, cubic GeTe possesses a lower formation energy for the Ge vacancy (negative value) when the Fermi level is around the VBM, in contrast to rhombohedral GeTe (slightly positive value). This means that Ge vacancies are more likely to spontaneously form in cubic $\mathrm{GeTe}$, independent of the growth environment (Ge environment or Te environment) and Fermi energy. This is consistent with the theoretical predictions of an increased Ge vacancy concentration with the working temperature ${ }^{23}$.

In principle, the presence of Ge vacancies in the GeTe system should strongly affect the structural stability and transport properties. The common view of the GeTe 

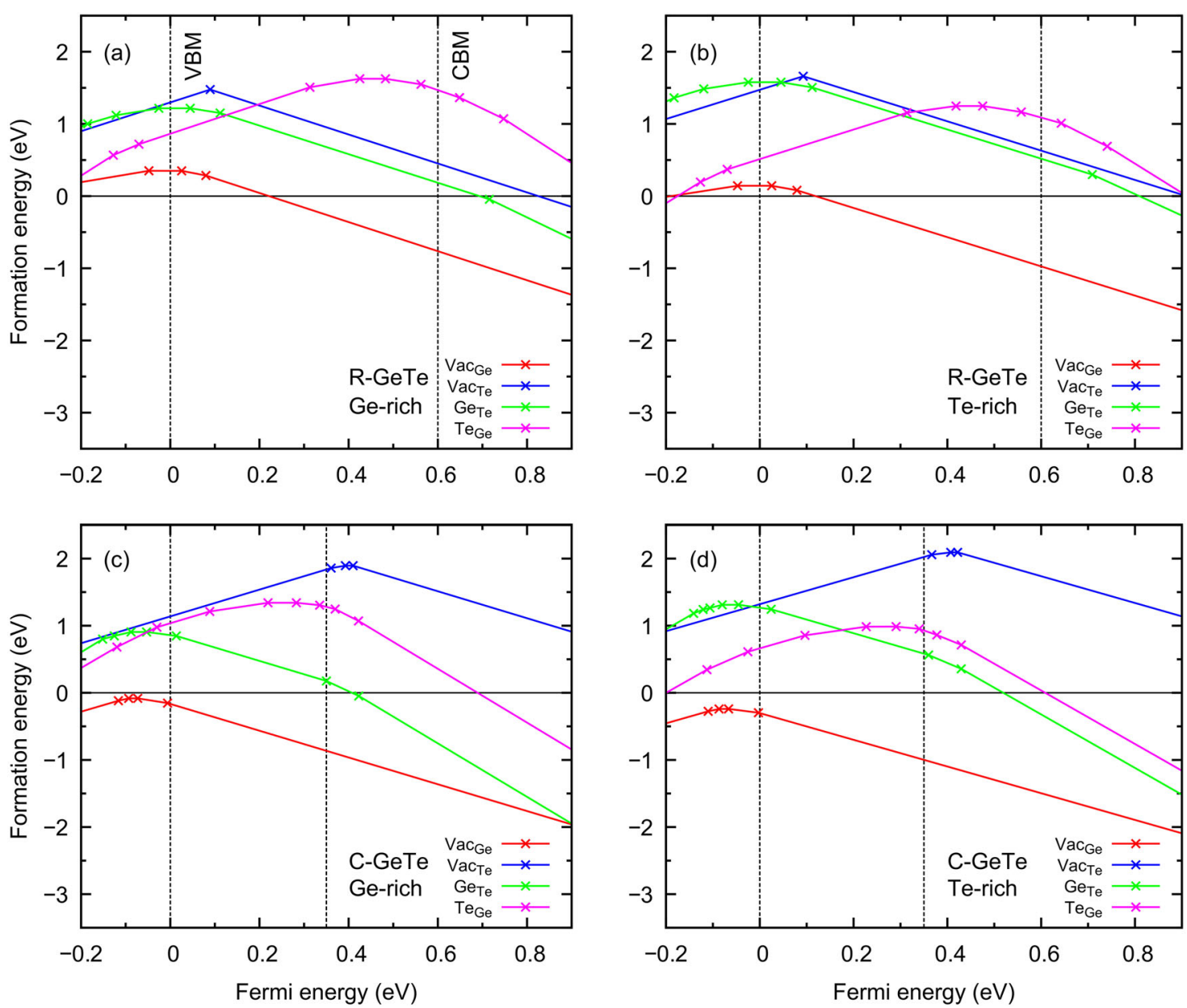

Fig. 1 Calculated defect formation energies of the low-energy intrinsic defects in rhombohedral GeTe (R-GeTe) and cubic GeTe (C-GeTe) as a function of Fermi energy in Ge-rich and Te-rich environments. $\mathbf{a}$, b Ge-rich and Te-rich environments for R-GeTe, respectively; $\mathbf{c}$, $\mathbf{d}$ Ge-rich and Te-rich environments for C-GeTe, respectively. The Fermi energy is referenced to the valence band maximum, and the vertical dashed black line indicates the band gap region within the GGA-PBE level.

system is that cubic GeTe, in theory, possesses better thermoelectric performance than rhombohedral $\mathrm{GeTe}^{31,37}$. In the following, the effect of Ge vacancies on the structure-property relationship in cubic GeTe is thoroughly investigated by combining DFT calculations and the Boltzmann transport equation.

First, calculations of the phonon dispersion and phonon density of states are performed to reveal the dynamical stability. The appearance of imaginary frequencies in the phonon dispersion of cubic GeTe in Fig. 2a indicates its thermodynamically unstable character at $0 \mathrm{~K}$. Although defective cubic GeTe with Ge vacancies $\left(\mathrm{C}-\mathrm{Ge}_{26} \mathrm{Te}_{27}\right.$ and $\left.\mathrm{C}-\mathrm{Ge}_{22} \mathrm{Te}_{27}\right)$ maintains the negative frequency in the phonon DOS, the upward frequency shift of the optical phonon modes means that the soft optical modes that appear in cubic GeTe become harder when adding Ge vacancies, as shown in Fig. 2 b. Similar results are also observed for $\mathrm{C}-\mathrm{Ge}_{25} \mathrm{Te}_{27}, \mathrm{C}-\mathrm{Ge}_{24} \mathrm{Te}_{27}$, and $\mathrm{C}-\mathrm{Ge}_{23} \mathrm{Te}_{27}$
(Supplementary Fig. S2). This suggests that defective cubic GeTe with Ge vacancies is more dynamically stable even though soft modes still exist.

Electronic transport calculations at different temperatures are performed by solving semiclassical Boltzmann transport equations within the rigid band and constant relaxation time approximations without considering the effect of carrier-vacancy scattering. Figure $3 a-c$ shows the corresponding calculation results of the electrical conductivity over the relaxation time $\sigma / \tau_{\text {ele }}$, Seebeck coefficient $S$, and power factor over the relaxation time $\mathrm{PF} / \tau_{\text {ele }}$ at $300 \mathrm{~K}$, respectively. It is revealed that cubic GeTe (C$\left.\mathrm{Ge}_{27} \mathrm{Te}_{27}\right)$ shows higher $\sigma / \tau_{\text {ele }}$, smaller $S$, and higher $\mathrm{PF} /$ $\tau_{\text {ele }}$ than rhombohedral GeTe ( $\left.\mathrm{R}_{-\mathrm{Ge}_{27}} \mathrm{Te}_{27}\right)$. Xing et al. ${ }^{37}$ explained that cubic GeTe possesses a combination of light band and heavy band features, high valley degeneracy, and strong pocket anisotropy. More importantly, defective cubic GeTe with Ge vacancies $\left(\mathrm{C}-\mathrm{Ge}_{26} \mathrm{Te}_{27}\right.$ and 

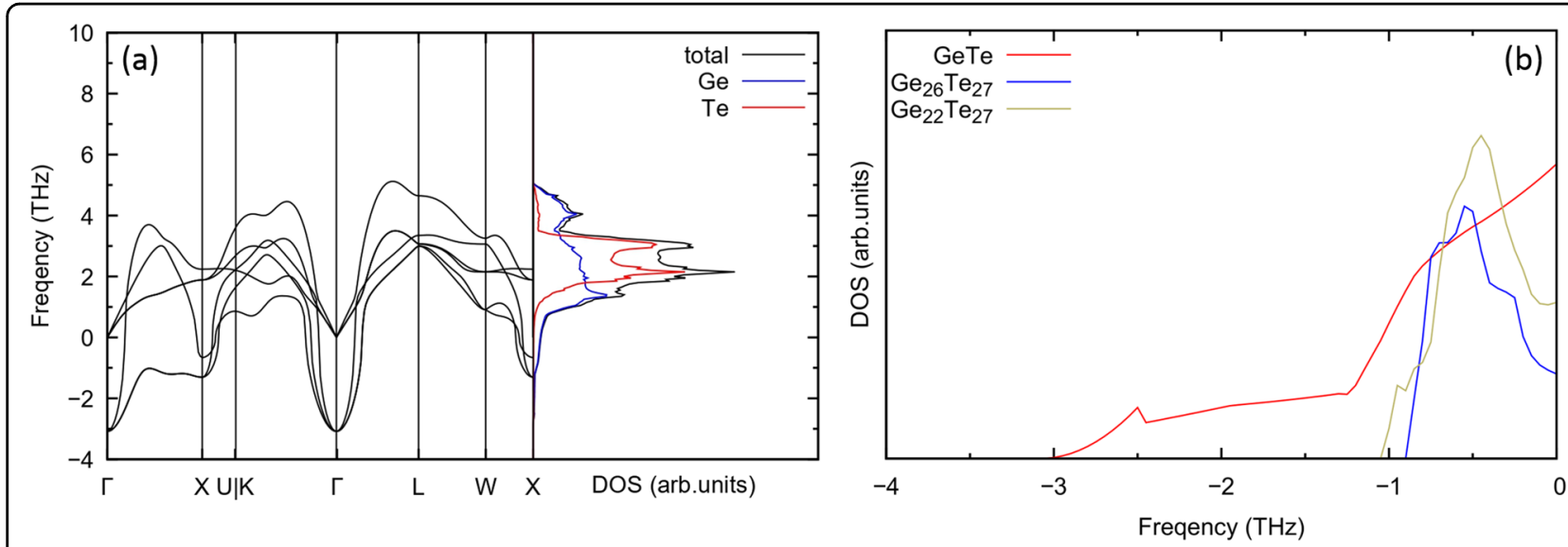

Fig. 2 Phonon dynamics of cubic GeTe with different concentrations of Ge vacancies. a Phonon dispersion and phonon density of states (DOS) of perfect cubic GeTe; $\mathbf{b}$ enlarged phonon DOS of cubic GeTe with different concentrations of Ge vacancies.

C- $\mathrm{Ge}_{22} \mathrm{Te}_{27}$ ) shows lower $\mathrm{PF} / \tau_{\text {ele }}$ than perfect cubic GeTe. This demonstrates that the introduction of Ge vacancies into cubic GeTe has a detrimental influence on the electronic valence band structure, rather than a beneficial optimization of the hole concentration. The declining tendency is also observed for $\mathrm{C}-\mathrm{Ge}_{25} \mathrm{Te}_{27}, \mathrm{C}-\mathrm{Ge}_{24} \mathrm{Te}_{27}$, and $\mathrm{C}-\mathrm{Ge}_{23} \mathrm{Te}_{27}$ (Supplementary Fig. S3). The calculated electrical transport properties at $600 \mathrm{~K}$ before the onset of the bipolar effect lead to the same conclusion (Supplementary Fig. S4). The calculated unfolded electronic band structures of the cubic supercell and defective cubic GeTe $\left(\mathrm{C}-\mathrm{Ge}_{26} \mathrm{Te}_{27}\right)$ are shown in Fig. 3d, e. After introducing Ge vacancies into cubic GeTe, the most obvious change is the reduced Fermi level, which is located inside the valence band, consistent with the calculated electronic DOS (Supplementary Fig. S5). This indicates a largely increased hole concentration in the defective cubic GeTe. Moreover, the increased carrier effective mass and band gap lead to an obviously decreased electrical conductivity in the Boltzmann transport calculation results. In addition, the position of the valence band at the $\Sigma$ point between $\mathrm{K}$ and $\Gamma$ is lifted up in the defective cubic GeTe. Because the valley degeneracy of the $\Sigma$ band is much higher than that of the L band ( 8 vs 4 ), the larger contribution of bands with high degeneracy results in a largely increased effective mass and thereby a higher Seebeck coefficient when the chemical potential is located near the band edge. Other defective cubic GeTe materials $\left(\mathrm{C}-\mathrm{Ge}_{25} \mathrm{Te}_{27}, \mathrm{C}\right.$ $\mathrm{Ge}_{24} \mathrm{Te}_{27}, \mathrm{C}-\mathrm{Ge}_{23} \mathrm{Te}_{27}$, and $\mathrm{C}-\mathrm{Ge}_{22} \mathrm{Te}_{27}$ ) show the same characteristics (Supplementary Fig. S6). The band structure calculations explicitly elucidate the previous Boltzmann transport calculation results.

It should be mentioned that the employment of appropriate carrier-vacancy scattering models in the future calculation process will further deteriorate the electrical transport properties of defective cubic GeTe with Ge vacancies owing to the reduction of $\sigma / \tau_{\text {ele }}$. Because the majority of previous investigations on cubic GeTe neglected the influence of Ge vacancies on performance optimization, the present theoretical calculations provide a real picture of the underlying mechanism in the GeTe system. The following experimental results of the symmetry change from rhombohedral approaching cubic and related property studies provide powerful evidence for our new perspective.

Here, we first found an interesting crystal structure evolution in which tetragonal $\mathrm{AgInTe}_{2}$ alloying in rhombohedral GeTe leads to the formation of cubic GeTe-based materials, as illustrated in the schematic diagram in Fig. 4a. This is demonstrated by the room-temperature X-ray diffraction (XRD) patterns of $(1-x) \mathrm{GeTe}+\mathrm{xAgInTe} \mathrm{e}_{2}$ samples $(x=0,0.01,0.02,0.03,0.04,0.05$, and 0.10; Fig. 4b). Below the critical AgInTe $\mathrm{Al}_{2}$ alloying content of $\sim 0.05$, the material structure exhibits a good match with rhombohedral GeTe, while a higher alloying content leads to the formation of a cubic structure. Further Rietveld refinement of the XRD patterns reveals that the calculated lattice parameters gradually decrease and the interaxial angles change from non$90^{\circ}$ to $90^{\circ}$ after $\operatorname{AgInTe} e_{2}$ alloying (Fig. 4c). This gives solid evidence for the structural evolution from a slightly distorted rock-salt lattice to a rock-salt lattice. In contrast, for single-element doping, including Ag doping and In doping, the structure remains rhombohedral, while $\mathrm{CuInTe}_{2}$ alloying results in the formation of a composite structure between rhombohedral GeTe and tetragonal $\mathrm{CuInTe}_{2}$ (Supplementary Fig. S7). Intuitively, considering the valence electron configuration of $\mathrm{Ge}, \mathrm{Ag}$, and In atoms, $\mathrm{Ag}$ and In codoping on the Ge site of the GeTe lattice should have an isoelectronic effect without obviously changing the hole concentration. In fact, AgInTe $\mathrm{I}_{2}$ alloying leads to a significant increase in the hole concentration, from $8.6 \times 10^{20} \mathrm{~cm}^{-3}$ to $1.25 \times 10^{22} \mathrm{~cm}^{-3}$, similar to Mn alloying (Fig. $\left.4 \mathrm{~d}\right)^{49,50}$. This 

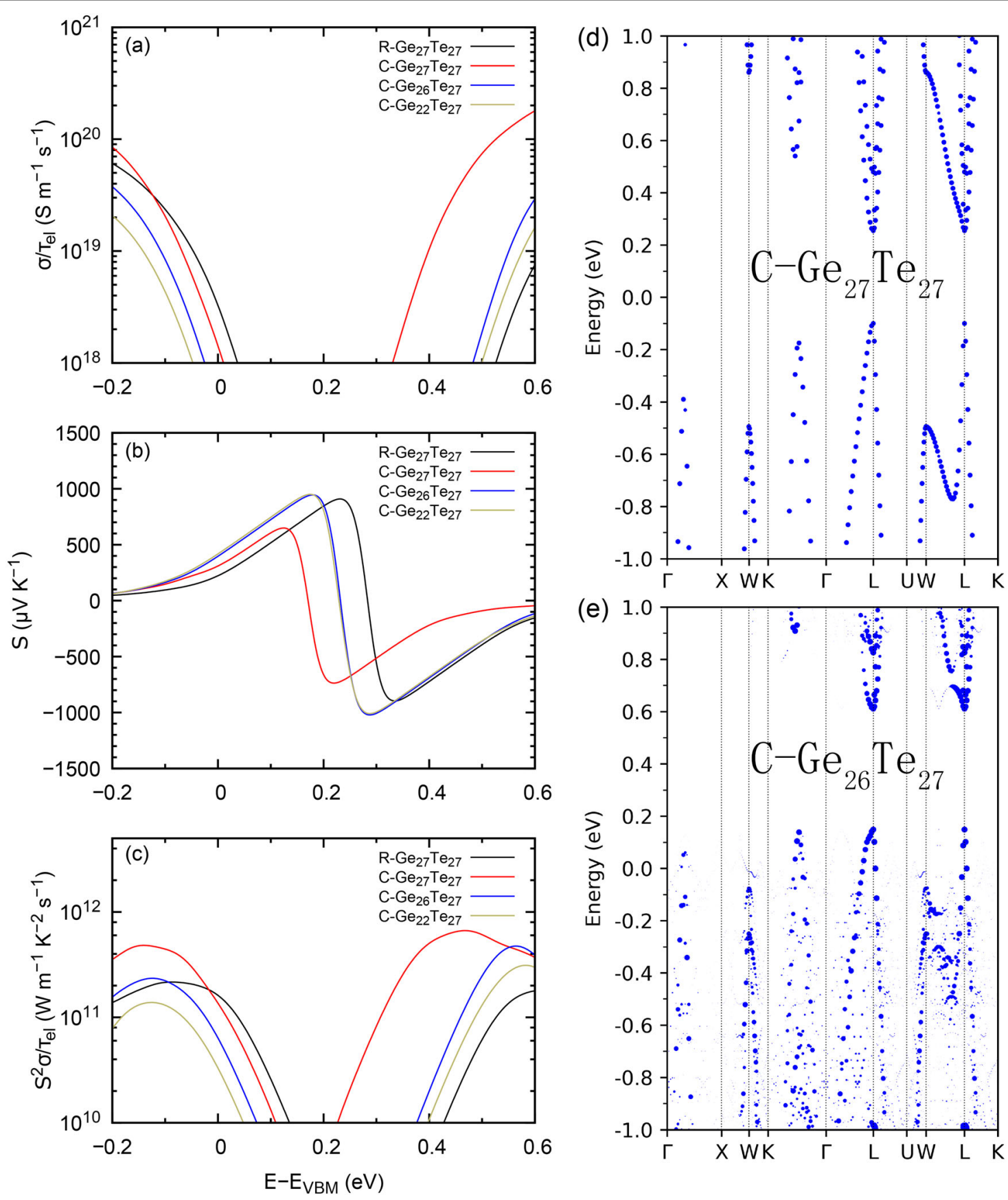

Fig. 3 DFT calculations about the role of Ge vacancy in cubic GeTe. a-c Theoretical electrical conductivity over relaxation time $\sigma / \tau_{\text {ele, }}$ Seebeck coefficient $\mathrm{S}$, and power factor over relaxation time $P F / \tau_{\text {ele }}$ at $300 \mathrm{~K}$ as a function of Fermi energy for rhombohedral GeTe $\left(\mathrm{R}_{-}-\mathrm{Ge}_{27} \mathrm{Te} \mathrm{e}_{27}\right), \mathrm{Cubic} \mathrm{GeTe}(\mathrm{C}-$ $\left.\mathrm{Ge}_{27} T e_{27}\right)$, and defective $\mathrm{C}-\mathrm{GeTe}$ with $\mathrm{Ge}$ vacancies $\left(\mathrm{C}-\mathrm{Ge}_{26} \mathrm{Te}_{27}\right.$ and $\left.\mathrm{C}-\mathrm{Ge}_{22} \mathrm{Te}_{27}\right)$, respectively. $\mathbf{d}$, e Calculated unfolded electronic band structures of $\mathrm{C}-\mathrm{Ge}_{27} \mathrm{Te}_{27}$ and $\mathrm{C}-\mathrm{Ge}_{26} \mathrm{Te}_{27}$, respectively. The plot size in the band structure reflects the unfolded spectral weight.

large difference is attributed to the increased concentration of Ge vacancies by AgInTe $e_{2}$ alloying or Mn alloying ${ }^{49,50}$, as a Ge vacancy contributes two holes to the valence band and thus sharply increases the hole concentration. In addition, we also synthesized two samples with nominal compositions of $\left(\mathrm{Ge}_{0.9} \mathrm{Te}\right)_{0.9}\left(\mathrm{AgInTe}_{2}\right)_{0.1}$ and $\left(\mathrm{Ge}_{0.95} \mathrm{Te}\right)_{0.9}\left(\mathrm{AgInTe}_{2}\right)_{0.1}$ to further confirm our conclusion. Reducing the Ge content leads to the appearance of impurity phase $\mathrm{Ag}_{2} \mathrm{Te}$, as shown in their XRD patterns (Supplementary Fig. S8), which indicates that the parent material $(\mathrm{GeTe})_{0.9}\left(\mathrm{AgInTe}_{2}\right)_{0.1}$ already contains a large amount of Ge vacancies. Therefore, the continued introduction of $\mathrm{Ge}$ vacancies into this 

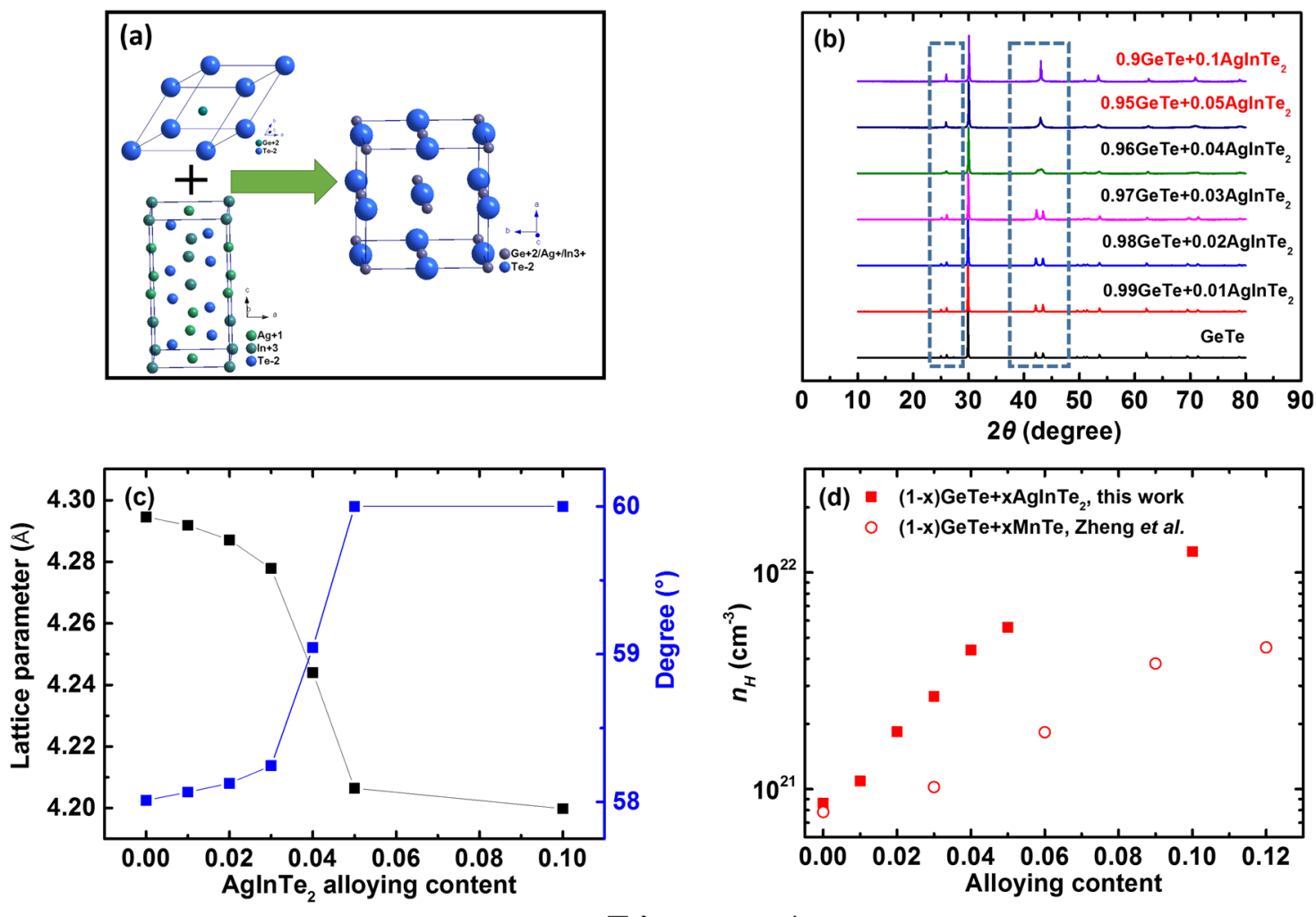

Fig. 4 Structural evolution in alloying GeTe with AgInTe 2 . a Schematic diagram of the crystal structure evolution in alloying rhombohedral GeTe and tetragonal AgInTe 2 to form cubic GeTe-based materials; b XRD patterns of $(1-x)$ GeTe+xAgInTe 2 samples $(x=0,0.01,0.02,0.03,0.04,0.05$, and 0.10); c lattice parameters and interaxial angles as a function of AglnTe $e_{2}$ alloying content; $\mathbf{d}$ hole concentration $n_{H}$ as a function of alloying content, including (1-x)GeTe+xAglnTe 2 and $(1-x) G e T e+x M n T e^{40}$.

structure would result in structural instability and simultaneously the appearance of a Te-rich impurity phase. The high Hall carrier concentration is almost unchanged (Supplementary Fig. S9), demonstrating the existence of a large amount of Ge vacancies in the parent phase. However, it is difficult to determine the exact mechanism underlying the worsened electrical performance (Supplementary Fig. S9), which is probably due to the appearance of impurity phases and/or the effect of Ge vacancies. In other words, this result in turn proves the lower formation energy of Ge vacancies in cubic GeTe. Based on the previous calculations of the phonon stability, the stabilization of the metastable cubic structure after $\mathrm{AgInTe}_{2}$ alloying or Mn alloying at room temperature is ascribed to the manipulation of the $\mathrm{Ge}$ vacancy concentration.

To elucidate the extrinsic doping effects on the electronic structure from $\mathrm{Ag}$ and In in detail, the electronic unfolded band structure and DOS of the AgInTe $\mathrm{A}_{2}$-alloyed composition $\left(\mathrm{Ge}_{62} \mathrm{Ag}_{1} \mathrm{In}_{1} \mathrm{Te}_{64}\right)$ were calculated. Apparently, the obtained band structure is fairly similar to that of cubic defective GeTe with Ge vacancies (Fig. 5a), where the carrier pocket located between $\mathrm{K}$ and $\Gamma$ is lifted up and the band gap is enlarged. The appearance of impurity states in the band gap is due to the hybridized states among In-s, Ge-s, Ge-p, and Te-p (Fig. 5b). Importantly, the Fermi level is located near the impurity states inside the band gap, which is significantly different from the defective cubic GeTe case where the Fermi level lies deeply in the valence band. Thus, it can be concluded that $\mathrm{Ag}$ and In do not act as acceptors, and additional Ge vacancies are introduced into our $\mathrm{AgInTe}_{2}$-alloyed samples.

Figure 6 shows the influence of $\mathrm{AgInTe}_{2}$ alloying on the thermoelectric properties of (1-x)GeTe+xAgInTe 2 . Due to the largely increased hole concentration arising from $\mathrm{AgInTe}_{2}$ alloying of more than one order, the carrier mobility $\mu_{\mathrm{H}}$ decreases from $56.0 \mathrm{~cm}^{2} \mathrm{~V}^{-1} \mathrm{~s}^{-1}$ to $1.6 \mathrm{~cm}^{2}$ $\mathrm{V}^{-1} \mathrm{~s}^{-1}$ (Fig. 6a). Basically, the AgInTe $\mathrm{A}_{2}$-alloyed samples possess $\mu_{\mathrm{H}}$ comparable or very close to those of $\mathrm{Mn}$ alloyed samples in the same range of $n_{H}$. Both of these mobilities, however, are lower than those of conventional GeTe-based samples (solid line). The extra carrier scattering mechanism arising from Ge vacancies existing in both $\mathrm{AgInTe}_{2}$-alloyed and $\mathrm{Mn}$-alloyed samples is responsible for the suppression of $\mu_{\mathrm{H}}$, which is revealed by the changed temperature-range dependence of the electrical 

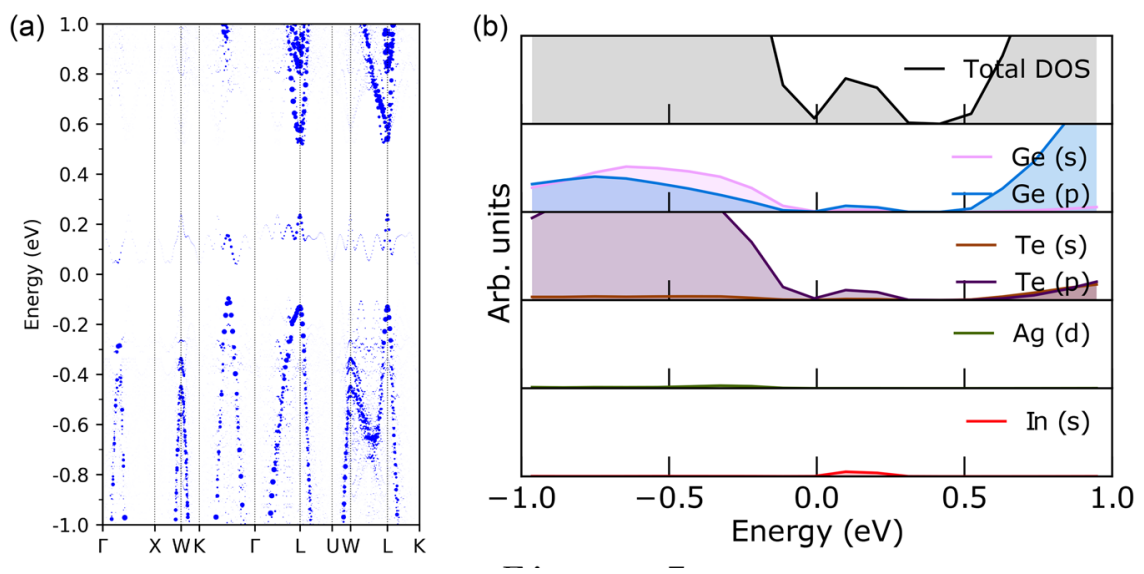

Fig. 5 Electronic band structrue and DOS after AgInTe 2 alloying. $\mathbf{a}, \mathbf{b}$ Unfolded band structure and total and partial density of states (DOS) of AglnTe -alloyed cubic GeTe $\left(\mathrm{Ge}_{62} \mathrm{Ag}_{1} \ln _{1} \mathrm{Te}_{64}\right)$, respectively. The plot size in the band structure reflects the unfolded spectral weight.

resistivity $\rho$. The $\rho$ below $600 \mathrm{~K}$ monotonically increases with increasing alloying content of $\mathrm{AgInTe}_{2}$ (Fig. 6b) due to the decreased $\mu_{\mathrm{H}}$, e.g., the room-temperature $\rho$ increases from $0.13 \times 10^{-5} \Omega \mathrm{m}$ to $0.32 \times 10^{-5} \Omega \mathrm{m}$. Meanwhile, it is observed that the temperature-range dependence of $\rho$ is dramatically changed, with the temperature exponent decreasing from 1.5 for GeTe down to 0.2 for $0.95 \mathrm{GeTe}-0.05 \mathrm{AgInTe}_{2}$. This is indicative of the carrier scattering mechanism changing from acoustic phonon scattering to mixed acoustic phonon and ionized impurity scattering after $\mathrm{AgInTe}_{2}$ alloying. This proves that the introduced large amount of $\mathrm{Ge}$ vacancies changes the carrier scattering mechanism. Despite the largely increased $n_{\mathrm{H}}$, the Seebeck coefficient $S$ in the lowtemperature range increases somewhat after $\mathrm{AgInTe}_{2}$ alloying, e.g., the room-temperature $S$ increases from $36.6 \mu \mathrm{V} \mathrm{K}^{-1}$ to $46.7 \mu \mathrm{V} \mathrm{K}^{-1}$. (Fig. 6c). Taking the increased $n_{\mathrm{H}}$ into consideration, this means that $\mathrm{AgInTe}_{2}$ alloying leads to a heavier character of the valence band structure. This is demonstrated by the calculated Pisarenko plot, showing the relationship between $S$ and $n_{\mathrm{H}}$ (Supplementary Fig. S10). As a result of the high $n_{\mathrm{H}}$ being out of the range of the optimized value and the reduced $\mu_{\mathrm{H}}$, the power factor $P F$ decreases after AgInTe $\mathrm{e}_{2}$ alloying (Fig. 6d). For example, the room-temperature $P F$ decreases from $10.4 \mu \mathrm{W} \mathrm{cm}{ }^{-1} \mathrm{~K}^{-2}$ for $\mathrm{GeTe}$ to $7.0 \mu \mathrm{W} \mathrm{cm} \mathrm{c}^{-1} \mathrm{~K}^{-2}$ for $0.95 \mathrm{GeTe}-0.05 \mathrm{AgInTe}_{2}$. The deteriorated $P F$ when the structure changes from rhombohedral to cubic is in good agreement with the results above based on the Boltzmann transport theory. The total thermal conductivity $\kappa_{\text {tot }}$ shows a sharp reduction after $\mathrm{AgInTe}_{2}$ alloying (Fig. 6e). After excluding the electronic thermal conductivity $\kappa_{\text {ele }}$ from $\kappa_{\text {tot}}$, it is found that the lattice thermal conductivity $\kappa_{\text {lat }}$ is significantly suppressed (Supplementary Fig. S11), e.g., the room-temperature $\kappa_{\text {lat }}$ decreases from $1.6 \mathrm{~W} \mathrm{~m}^{-1} \mathrm{~K}^{-1}$ to $0.5 \mathrm{~W} \mathrm{~m}^{-1} \mathrm{~K}^{-1}$. Moreover, the $\kappa_{\text {lat }}$ values of $\mathrm{AgInTe}_{2}$ - alloyed samples are seriously overestimated based on the Debye-Callaway model (the inset of Fig. 6e). This anomalous suppression of $\kappa_{\text {lat }}$ is attributed to the strong phonon scattering due to the formation of Ge vacancies. Finally, the peak $Z T$ gradually decreases as the $\mathrm{AgInTe}_{2}$ alloying content increases (Fig. 6f).

Although defective cubic GeTe may not be a good platform for achieving high p-type performance, the large number of Ge vacancies in the structure act as suitable sites for foreign atoms and therefore enable high dopant solubility. This can provide hints for realizing the sought-after n-type conduction in the GeTe system. Bismuth (Bi) is an effective dopant in the GeTe system for optimizing the hole concentration. However, due to the limited solubility, heavy $\mathrm{Bi}$ doping leads to the appearance of impurity phases. XRD measurement of the sample with the nominal composition $\mathrm{Ge}_{0.8} \mathrm{Bi}_{0.2} \mathrm{Te}$ confirms the impurity phases as $\mathrm{Bi}_{2} \mathrm{Ge}_{3} \mathrm{Te}_{6}$ and $\mathrm{Bi}_{4} \mathrm{Te}_{3}$, while the sample with the nominal composition $\left.(0.95 \mathrm{GeTe}+0.05 \mathrm{AgInTe})_{2.8}\right)_{0.2} \mathrm{Bi}_{0.2} \mathrm{Te}$ shows a single-phase character (Fig. 7a). The vanishing of impurity phases is possibly due to the high concentration of Ge vacancies, as mentioned before. More importantly, the former sample shows unstable $n$-type conduction during the thermal cycling process. The electrical resistivity $\rho$ increases and simultaneously the Seebeck coefficient $S$ decreases (Figs. 7b, c), which indicates that the n-type conduction becomes weakened and may finally vanish after a long-term thermal cycling test. In sharp contrast, the latter sample exhibits lower $\rho$ and higher $S$, which accounts for its n-type conduction behavior being stronger than the former (Figs. 7b, c). In addition, the almost unchanged transport data definitely confirm the stable $n$-type conduction against thermal cycling, meaning achievement of good structural stability after Bi heavy doping. 

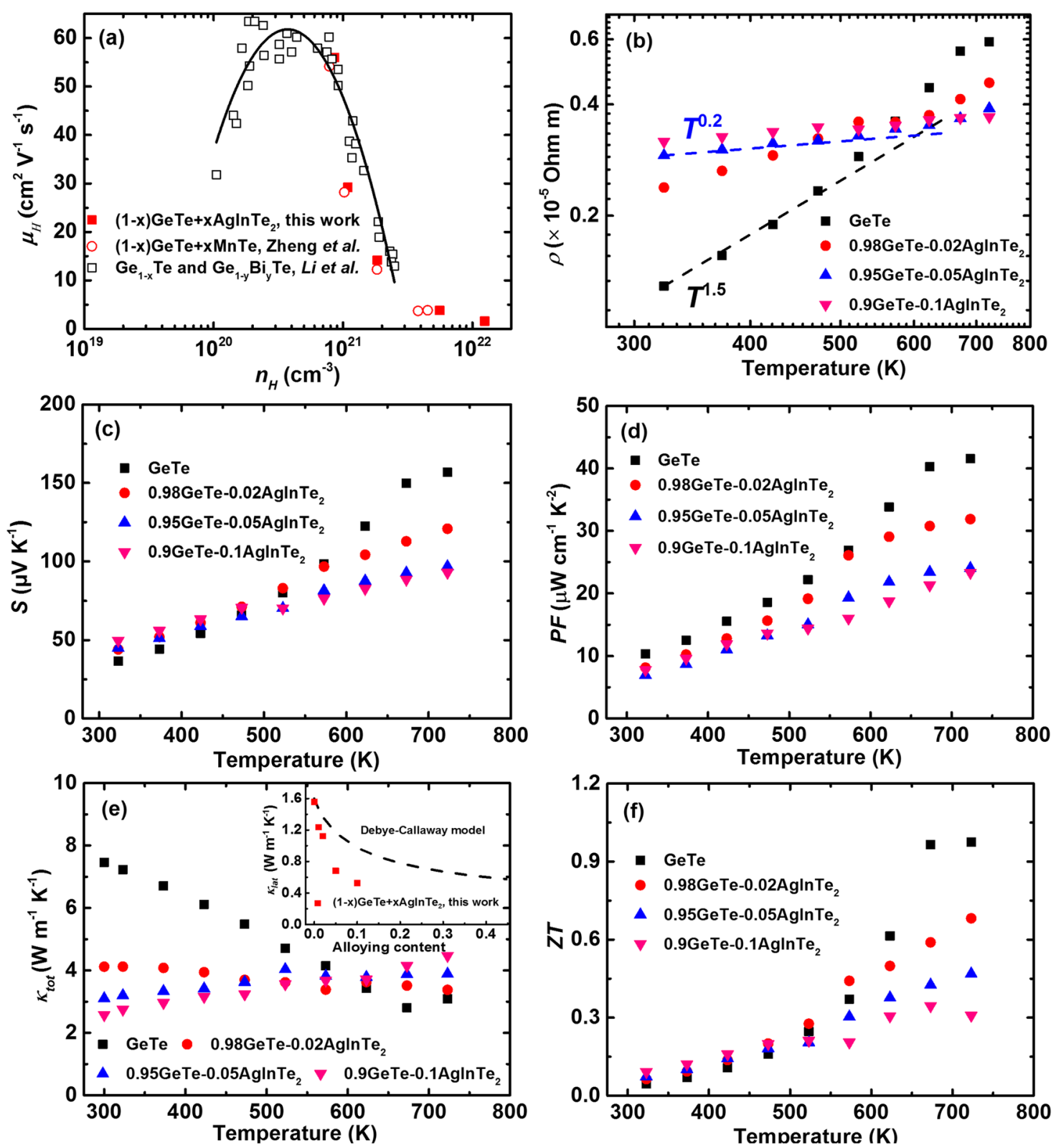

Fig. 6 Influence of AgInTe $e_{2}$ alloying on the thermoelectric properties of (1-x)GeTe $+x A g \operatorname{lnTe}(x=0,0.02,0.05$, and 0.1$)$. a Carrier mobility $\mu_{H}$ as a function of hole concentration $n_{H}$ in comparison to $(1-x) \mathrm{GeTe}+x M n T e^{40}$, as well as $\mathrm{Ge}_{1+x} \mathrm{Te}$ and $\mathrm{Ge}_{1-y} \mathrm{Bi}_{y} \mathrm{Te}^{27}$. The solid line is included as a guide to the eye. Temperature-dependent electrical resistivity $\rho(\mathbf{b})$, Seebeck coefficient $S(\mathbf{c})$, power factor PF $(\mathbf{d})$, total thermal conductivity $K_{\text {tot }}(\mathbf{e})$, and ZT (f). The inset in $\mathbf{e}$ shows the room-temperature lattice thermal conductivity $k_{\text {lat }}$ as a function of alloying content, where the dashed line represents the theoretical $k_{\text {lat }}$ based on the Debye-Callaway model.

\section{Conclusions}

In summary, we reshaped the role of Ge vacancies in the crystal structure-thermoelectric property relationship in the GeTe system by combining first-principles calculations and Boltzmann transport theory. It is concluded that $\mathrm{Ge}$ vacancies behave as the most easily formed point defect, leading to stabilization of the metastable cubic structure, and show a detrimental influence on the electrical transport properties.
Experimental investigations found that $\mathrm{AgInTe}_{2}$ alloying promoted a symmetry change from rhombohedral to cubic and deteriorated the thermoelectric performance, proving our previous theoretical calculations. Owing to the deep understanding of defect chemistry, a singlephase cubic GeTe-based material with stable n-type conduction was discovered, which may open the door for future studies of thermoelectric systems with asymmetrical performance. 

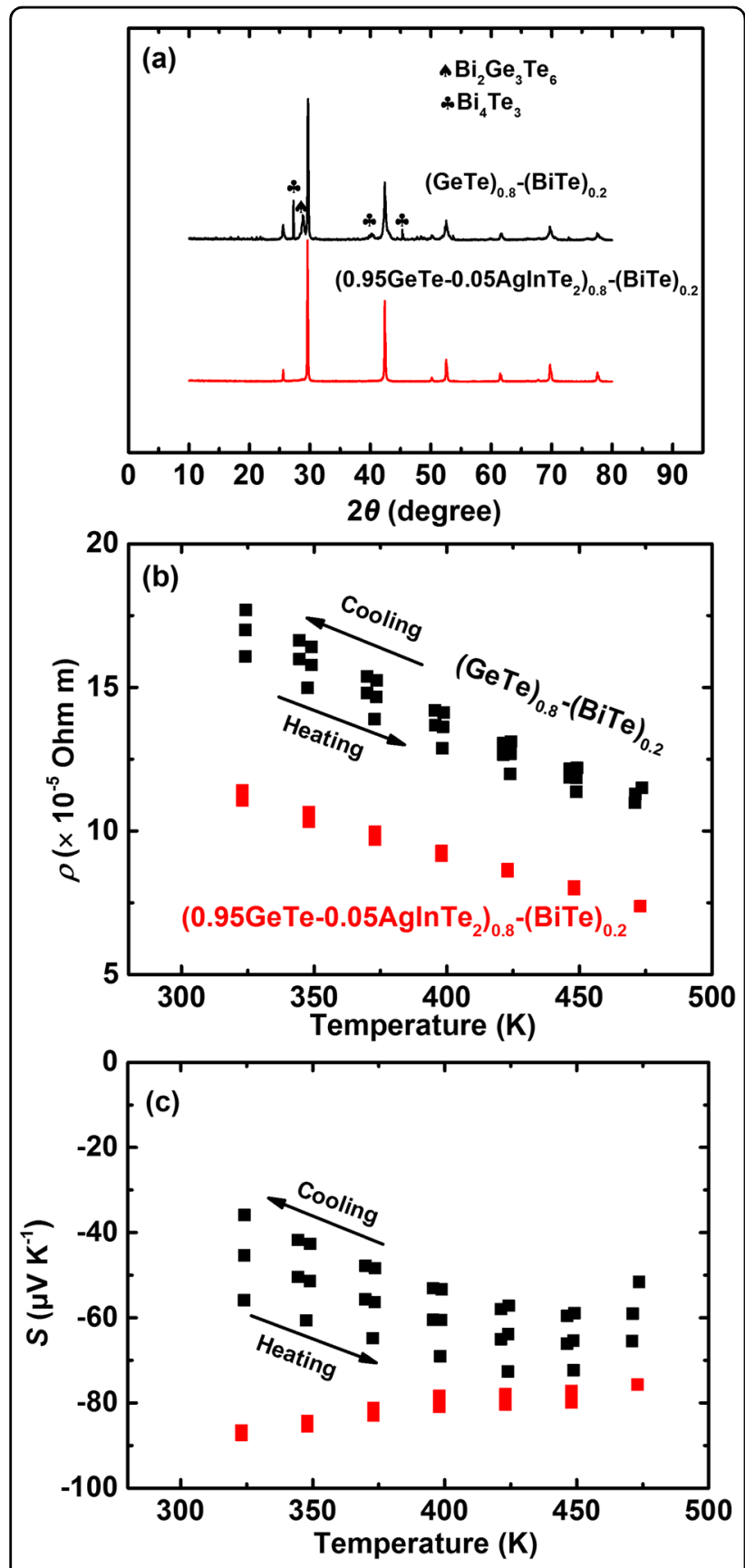

Fig. 7 Comparison of the structure and n-type conduction behavior of $\mathrm{Bi}$ heavily doped samples $\left(\mathrm{Ge}_{0.8} \mathrm{Bi}_{0.2} \mathrm{Te}\right.$ vs $(0.95 \mathrm{GeTe}$ $\left.+\mathbf{0 . 0 5} \mathrm{Ag}_{\mathrm{InTe}}\right)_{0.8} \mathrm{Bi}_{0.2} \mathrm{Te}$ ). a XRD patterns before the thermal cycling measurement; temperature-dependent electrical resistivity $\rho(\mathbf{b})$ and Seebeck coefficient S (c) during thermal cycling.

\section{Acknowledgements}

This work was supported by JST Mirai Program Grant Number JPMJMI19A1, CREST JPMJCR19Q4, and JSPS KAKENHI JP17H02749, JP16H06441. The computation in this work was performed using the Numerical Materials Simulator at NIMS and SGI Rackable C2112-4GP3/C1102-GP8 (Reedbush-U/H/L) in the Information Technology Center, The University of Tokyo. Z.L. acknowledges the financial support from the NIMS-MANA Postdoctoral Fellowship.
Conflict of interest

The authors declare that they have no conflict of interest.

\section{Publisher's note}

Springer Nature remains neutral with regard to jurisdictional claims in published maps and institutional affiliations.

Supplementary information is available for this paper at https://doi.org/ 10.1038/s41427-020-00247-y

Received: 25 April 2020 Revised: 15 July 2020 Accepted: 17 July 2020. Published online: 2 October 2020

\section{References}

1. Petsagkourakis, I. et al. Thermoelectric materials and applications for energy harvesting power generation. Sci. Technol. Adv. Mater. 19, 836-862 (2018).

2. Shi, X., Chen, L. \& Uher, C. Recent advances in high-performance bulk thermoelectric materials. Int. Mater. Rev. 61, 379-415 (2016).

3. Mao, J. et al. Advances in thermoelectrics. Adv. Phys. 67, 69-147 (2018).

4. Mori, T. Novel principles and nanostructuring methods for enhanced thermoelectrics. Small 13, 1702013 (2017).

5. Pei, Y. Z. et al. Convergence of electronic bands for high performance bulk thermoelectrics. Nature 473, 66-69 (2011)

6. Yang, J. et al. On the tuning of electrical and thermal transport in thermoelectrics: an integrated theory-experiment perspective. npj Comput. Mater. 2, 15015 (2016).

7. Liu, Z. H., Mao, J., Liu, T.-H., Chen, G. \& Ren, Z. F. Nano-microstructural control of phonon engineering for thermoelectric energy harvesting. MRS Bull. 43, 181-186 (2018)

8. Khan, A. U. et al. Nano-micro-porous skutterudites with $100 \%$ enhancement in ZT for high performance thermoelectricity. Nano Energy 31, 152-159 (2017).

9. Zhao, L.-D. et al. Ultralow thermal conductivity and high thermoelectric figure of merit in SnSe crystals. Nature 508, 373-377 (2014).

10. Chang, C. \& Zhao, L.-D. Anharmoncity and low thermal conductivity in thermoelectrics. Mater. Today Phys. 4, 50-57 (2018).

11. Zhao, W. Y. et al. Magnetoelectric interaction and transport behaviours in magnetic nanocomposite thermoelectric materials. Nat. Nanotechnol. 12, 55 (2017).

12. Vaney, J.B. et al. Magnetism-mediated thermoelectric performance of the $\mathrm{Cr}$ doped bismuth telluride tetradymite. Mater. Today Phys. 9, 100090 (2019).

13. Zhu, H. T. et al. Discovery of TaFeSb-based half-Heuslers with high thermoelectric performance. Nat. Commun. 10, 270 (2019).

14. Pei, Y. Z. et al. Interstitial point defect scattering contributing to high thermoelectric performance in SnTe. Adv. Electron. Mater. 2, 1600019-n/a (2016).

15. You, L. et al. Boosting the thermoelectric performance of PbSe through dynamic doping and hierarchical phonon scattering. Energy Environ. Sci. 11, 1848-1858 (2018).

16. Tan, G. J. et al. High thermoelectric performance SnTe-In2Te3 solid solutions enabled by resonant levels and strong vacancy phonon scattering. Chem. Mater. 27, 7801-7811 (2015)

17. Mao, J. et al. Self-compensation induced vacancies for significant phonon scattering in InSb. Nano Energy 48, 189-196 (2018).

18. Liu, X. H. et al. Significant roles of intrinsic point defects in $\mathrm{Mg} 2 \mathrm{X}(\mathrm{X}=\mathrm{Si}, \mathrm{Ge}, \mathrm{Sn})$ thermoelectric materials. Adv. Electron. Mater. 2, 1500284 (2015).

19. Tamaki, H., Sato, H. K. \& Kanno, T. Isotropic conduction network and defect chemistry in Mg3+ 8 Sb2-based layered zintl compounds with high thermoelectric performance. Adv. Mater. 28, 10182-10187 (2016).

20. Gorai, P., Stevanović, V. \& Toberer, E. S. Computationally guided discovery of thermoelectric materials. Nat. Rev. Mater. 2, 17053 (2017).

21. Hu, L. P., Zhu, T. J., Liu, X. H. \& Zhao, X. B. Point defect engineering of highperformance bismuth-telluride-based thermoelectric materials. Adv. Funct. Mater. 24, 5211-5218 (2014).

22. Liu, Z. H. et al. Understanding and manipulating the intrinsic point defect in [small alpha]-MgAgSb for higher thermoelectric performance. J. Mater. Chem. A 4, 16834-16840 (2016)

23. Edwards, A. H. et al. Theory of persistent, p-type, metallic conduction in cGeTe. J. Phys. Condens. Matter 17, L329 (2005).

24. Zhang, W. et al. Role of vacancies in metal-insulator transitions of crystalline phase-change materials. Nat. Mater. 11, 952-956 (2012). 
25. Gelbstein, Y., Davidow, J., Girard, S. N., Chung, D. Y. \& Kanatzidis, M. Controlling metallurgical phase separation reactions of the $\mathrm{Ge} 0.87 \mathrm{Pb0}$ 13 Te alloy for high thermoelectric performance. Adv. Energy Mater. 3 815-820 (2013).

26. Wu, D. et al. Origin of the high performance in GeTe-based thermoelectric materials upon Bi2Te3 doping. J. Am. Chem. Soc. 136, 11412-11419 (2014).

27. Li, J. et al. Electronic origin of the high thermoelectric performance of GeTe among the p-type group IV monotellurides. NPG Asia Mater. 9, e353 (2017).

28. Zhang, $X$. et al. Vacancy manipulation for thermoelectric enhancements in GeTe alloys. J. Am. Chem. Soc. 140, 15883-15888 (2018).

29. Dong, J. et al. Medium-temperature thermoelectric GeTe: vacancy suppression and band structure engineering leading to high performance. Energy Environ. Sci. 12, 1396-1403 (2019).

30. Li, J. et al. Low-symmetry rhombohedral GeTe thermoelectrics. Joule 2 , 976-987 (2018).

31. Hong, M. et al. Realizing zT of 2.3 in Ge1-x-ySbxInyTe via reducing the phase-transition temperature and introducing resonant energy doping. Adv. Mater. 30, 1705942 (2018).

32. Xing, T. et al. Superior performance and high service stability for GeTe-based thermoelectric compounds. Natl Sci. Rev. 6, 944-954 (2019)

33. Perumal, S. et al. Realization of high thermoelectric figure of merit in GeTe by complementary co-doping of Bi and In. Joule 3, 2565-2580 (2019).

34. Jin, Y. et al. Realizing high thermoelectric performance in GeTe through optimizing Ge vacancies and manipulating Ge precipitates. ACS Appl. Energy Mater. 2, 7594-7601 (2019)

35. Shuai, J., Sun, Y., Tan, X. \& Mori, T. Manipulating the Ge vacancies and Ge precipitates through $\mathrm{Cr}$ doping for realizing the high-performance GeTe thermoelectric material. Small 16, 1906921 (2020).

36. Sist, M., Kasai, H., Hedegaard, E. M. \& Iversen, B. B. Role of vacancies in the hightemperature pseudodisplacive phase transition in GeTe. Phys. Rev. B 97, 094116 (2018)

37. Xing, G. et al. Thermoelectric properties of p-type cubic and rhombohedral GeTe. J. Appl. Phys. 123, 195105 (2018).
38. Shuai, J. et al. Enhanced thermoelectric performance through crystal field engineering in transition metal-doped GeTe. Mater. Today Phys. 9, 100094 (2019).

39. Samanta, M., Ghosh, T., Arora, R., Waghmare, U. V. \& Biswas, K. Realization of both n-and p-type GeTe thermoelectrics: electronic structure modulation by AgBiSe2 alloying. J. Am. Chem. Soc. 141, 19505-19512 (2019).

40. Kresse, G. \& Furthmüller, J. Efficiency of ab-initio total energy calculations for metals and semiconductors using a plane-wave basis set. Comput. Mater. Sci. 6, 15-50 (1996).

41. Perdew, J. P., Burke, K. \& Ernzerhof, M. Generalized gradient approximation made simple. Phys. Rev. Lett. 77, 3865 (1996).

42. Van De Walle, A. Multicomponent multisublattice alloys, nonconfigurational entropy and other additions to the Alloy Theoretic Automated Toolkit. Calphad 33, 266-278 (2009).

43. Monkhorst, H. J. \& Pack, J. D. Special points for Brillouin-zone integrations. Phys. Rev. B 13, 5188 (1976).

44. Popescu, V. \& Zunger, A. Extracting $E$ versus $\vec{k}$ effective band structure from supercell calculations on alloys and impurities. Phys. Rev. B 85, 085201 (2012).

45. Madsen, G. K. \& Singh, D. J. BoltzTraP. A code for calculating band-structure dependent quantities. Comput. Phys. Commun. 175, 67-71 (2006).

46. Togo, A. \& Tanaka, I. First principles phonon calculations in materials science. Scr. Mater. 108, 1-5 (2015).

47. Kumagai, Y. \& Oba, F. Electrostatics-based finite-size corrections for firstprinciples point defect calculations. Phys. Rev. B 89, 195205 (2014).

48. Broberg, D. et al. PyCDT: A Python toolkit for modeling point defects in semiconductors and insulators. Comput. Phys. Commun. 226, 165-179 (2018).

49. Zheng, Z. et al. Rhombohedral to cubic conversion of GeTe via MnTe alloying leads to ultralow thermal conductivity, electronic band convergence, and high thermoelectric performance. J. Am. Chem. Soc. 140 2673-2686 (2018).

50. Liu, Z. H. et al. Phase-transition temperature suppression to achieve cubic GeTe and high thermoelectric performance by Bi and Mn codoping. Proc. Natl Acad. Sci. USA 115, 5332-5337 (2018). 